\title{
Simulation of gas flow in microchannels with a sudden expansion or contraction
}

\author{
By AMIT AGRAWAL†, LYAZID DJENIDI \\ AND R. A. ANTONIA \\ Discipline of Mechanical Engineering, University of Newcastle, NSW 2308, Australia
}

(Received 15 December 2003 and in revised form 17 January 2005)

\begin{abstract}
Two-dimensional simulations based on the isothermal lattice-Boltzmann method have been undertaken on microchannels with a sudden expansion or contraction. The study provides insight into the analysis of flows in complicated microdevices. The flow is pressure driven, and computations are performed for several Knudsen numbers, and area and pressure ratios, allowing the effects of compressibility and rarefaction to be assessed. The pressure drop for both the converging and diverging channels shows a discontinuity in slope at the junction, and is accompanied by a jump in velocity. The pressure drop in each section can be predicted well by the theory for straight channels. The mass flow ratio between converging and diverging channels is close to unity, and the streamlines are attached in both cases. It is deduced that compressibility and rarefaction have opposite effects on the flow. These results suggest that complex channels of the type considered here can be understood in terms of their primary units, and they experience only small secondary losses.
\end{abstract}

\section{Introduction}

Micro-electro-mechanical systems (MEMS) devices with a typical length scale between $1 \mathrm{~mm}$ and 1 micron are smaller than objects commonly encountered, and exhibit several non-intuitive physical phenomena. They have a large surface-to-area ratio and therefore surface forces become important. The Knudsen number $(K n)$ is in the range of 0.001 to 0.1 , which corresponds to a regime whereby the no-slip boundary condition is violated at solid interfaces. The slip at the wall has to be correctly modelled before realistic theoretical and numerical results can be obtained. Not surprisingly, very little is known about the physics of these microdevices and data are scanty. Because MEMS has a potentially large number of engineering and biological applications (Ho \& Tai 1998; Beebe, Mensing \& Walker 2002), the last decade has seen a surge of research on these devices.

An understanding of fluid flow in microdevices is imperative before new and efficient micropumps and microactuators can be fabricated. Fluid flow in these devices is often through channels with changes in cross-sectional area, and/or channels with bends and bifurcations. A systematic study of these different configurations will provide valuable insight into the flow physics. The goal of the present study is to undertake a numerical study of diverging and converging channels with a sudden change in cross-sectional area in order to identify the factors affecting the flow, and to document

\footnotetext{
$\dagger$ Present address: Department of Mechanical Engineering, Indian Institute of Technology, Mumbai 400076, India.
} 
the differences between macro and micro scales for these geometries. The originality of this work is in the use of the lattice-Boltzmann method (LBM) which is perhaps better suited to microflows than the conventional Navier-Stokes solvers.

Flow through a straight channel is the simplest configuration where theoretical (Arkilic, Schmidt \& Breuer 1997), experimental (Pong et al. 1994; Meinhart, Wereley \& Santiago 2000) and numerical (Beskok, Karniadakis \& Trimmer 1996) results are available. Arkilic et al. (1997) solved the Navier-Stokes equations using perturbation methods and applied a slip boundary condition to obtain the variations in pressure, and longitudinal and lateral velocities in the channel. The analytical solution was verified against the experimental data of Pong et al. (1994) and Arkilic et al. (1997). Meinhart et al. (2000) used micro-PIV to measure the velocity field in straight channels. Beskok et al. (1996) performed numerical simulations to understand the effects of compressibility and rarefaction, and investigated the appropriate boundary conditions for the flow.

Flows through more complicated geometries have been studied experimentally by Lee, Wong \& Zohar (2002) and Li et al. (2000) using integrated pressure sensors. Lee et al. (2002) performed measurements in two channels connected in series with the connection between the channels having an included angle of $5^{\circ}, 15^{\circ}, 90^{\circ}$ or $180^{\circ}$. They measured the pressure drop and mass flow rate in the channel for $K n=0.06$ corresponding to nitrogen at atmospheric conditions. They found a large pressure drop at the intersection of the channels, and suggested replacing the junction with another channel with an equivalent pressure drop for the purpose of analysis. Li et al. (2000) compared flows through an orifice and a Venturi at small Reynolds number $(R e=0.05)$ and reported that flow separation can occur at the corners, with a vortex of 10 microns in diameter. In this paper, we present results from LBM simulations in two-dimensional microchannels of different widths connected in series with an included angle of $180^{\circ}$. Our computations are isothermal and therefore there is no temperature variation. The isothermal assumption is justified with silicon (which is commonly used for MEMS components) because it is a good heat conductor (Karniadakis \& Beskok 2002). The velocity and pressure variations and the resulting flow field are computed. The results suggest that the flow in (such) complex channels can be understood from a knowledge of the flow in a straight channel.

\section{Numerical procedure}

\subsection{The lattice-Boltzmann method}

LBM is based on the movement of a number of particles on a lattice (Frisch, Hasslacher \& Pomeau 1986). With sufficient symmetry of the lattice, the method inherently solves the compressible Navier-Stokes equations with second-order accuracy. Although a relatively new technique, LBM has been applied to diverse flow situations. Here, each computational node comprises eight moving particles and a rest particle (figure $1 a$ ). The particle density evolution equation is given by

$$
f_{i}\left(\boldsymbol{x}+\boldsymbol{e}_{i}, t+1\right)=f_{i}(\boldsymbol{x}, t)-\frac{f_{i}(\boldsymbol{x}, t)-f_{i}^{e q}(\boldsymbol{x}, t)}{\tau}
$$

where $f_{i}$ is the instantaneous particle density at a link, $\boldsymbol{e}_{i}$ are the direction vectors (figure $1 a$ ), $\tau$ is the relaxation time, and $f_{i}^{e q}$ is the corresponding equilibrium density computed as $f_{i}^{e q}=\rho w_{i}\left(1+3\left(\boldsymbol{e}_{i} \cdot \boldsymbol{u}\right)+\frac{9}{2}\left(\boldsymbol{e}_{i} \cdot \boldsymbol{u}\right)^{2}-\frac{3}{2} u^{2}\right)$. Here, $\boldsymbol{u}$ is the instantaneous velocity at the node, $\rho$ is the fluid density, and $w_{i}$ are the corresponding weights $\left(w_{i}=4 / 9\right.$ for particle $0,1 / 9$ for particles $1-4$, and $1 / 36$ for particles $\left.5-8\right)$. The 

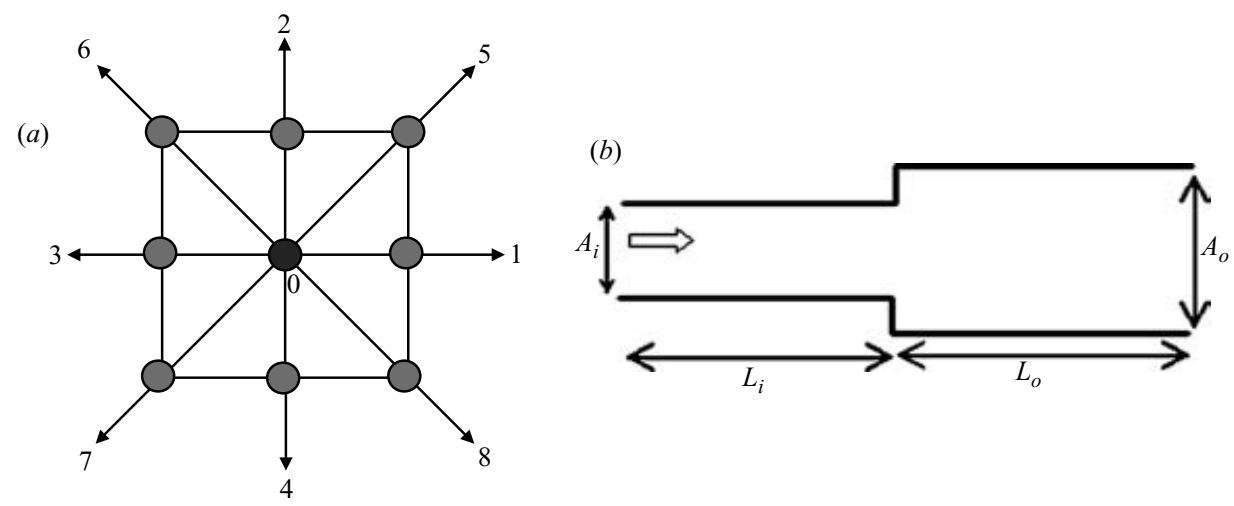

Figure 1. (a) Computational node. There are eight moving particles (denoted 1 to 8 ), and a particle at rest $(0)$. The direction of motion of a particle is indicated by the corresponding arrow. $(b)$ Computational domain. Ratio of inlet $\left(A_{i}\right)$ to outlet areas $\left(A_{o}\right)$ determines the area ratio of the microchannel. The arrow indicates the flow direction (which is left to right for the diverging channel and right to left for the converging channel).

relaxation time is related to the kinematic viscosity of the fluid via the relation $v=(2 \tau-1) / 6$. Equation $(2.1)$ is solved through the two steps of propagation and collision using a BGK collision operator (Chen \& Doolen 1998). The density and velocity of fluid at a node are calculated from the equations $\rho=\sum_{i} f_{i}, \rho \boldsymbol{u}=\sum_{i} f_{i} \boldsymbol{e}_{i}$. Given the nature of this method, the use of LBM to simulate flows at high $K n$ seems a natural choice.

Nie, Doolen \& Chen (2002) and Lim et al. (2002) have suggested modifications to the scheme in order to simulate high-Kn flows $(K n>0.001$ corresponding to the slip flow regime). The basic idea in both these approaches is to change the relaxation time appropriately. Nie et al. (2002) made $\tau$ a function of the density such that the dynamic viscosity remains constant, whereas Lim et al. (2002) made $\tau$ a function of $K n$. Either of these schemes is easy to implement in a standard LBM code. In the former approach, $K n$ is not known a priori, but the method is applicable to a wider range of $K n$. On the other hand, $K n$ can be fixed before the start of the computations in the latter approach, but the resolution depends on $K n$ and consequently a relatively small range of $K n$ can be investigated. Here we have adopted the scheme suggested by Nie et al. (2002) with $\tau^{\prime}=\frac{1}{2}+(1 / \rho)\left(\tau-\frac{1}{2}\right)$ where $\tau^{\prime}$ is the modified relaxation time. Note that this scheme is primarily valid for low Knudsen number which is the subject of investigation here.

\subsection{Computational details}

The computational domain consists of a two-dimensional channel with different widths (figure $1 b$ ). The narrower section is represented by 10 points, and the area ratio determines the number of points for the wider section (the area ratio is 2 for most of the computations reported here). The total number of points is 1000 in the streamwise direction with 500 points representing each of the narrow and wide sections. The length to width ratio is about 50 and 25 for the narrow and wide sections respectively, implying that the assumption of long channels is applicable here. The numerical resolution (of about $1000 \times 20$ ) appears to be sufficient for these computations. The extra computational points on the narrow section are prescribed as solid nodes and do not affect the flow. 

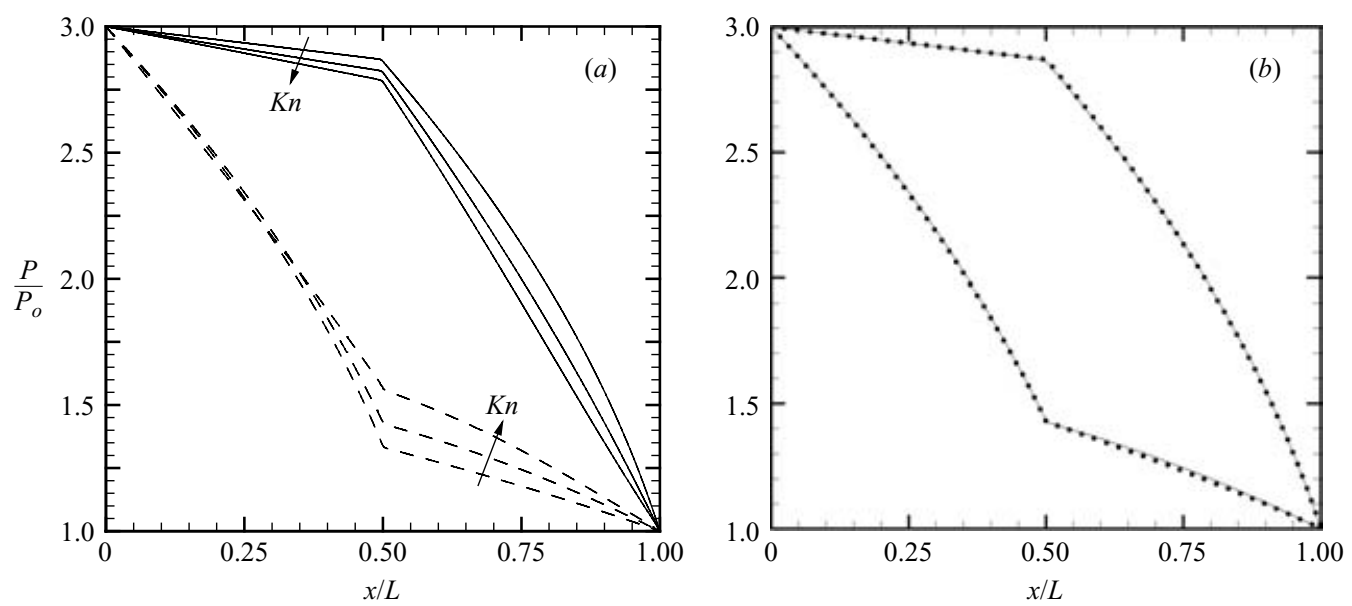

FIGURE 2. (a) Pressure distribution in converging (solid lines) and diverging channels (dashed lines) for different values of $K n$. The arrow is the direction of increasing $K n$. (b) Comparison of pressure between simulations (line) and theory (dots) for the converging (top curve) and diverging (bottom curve) channels. The Knudsen numbers at the outlet are 0.012 and 0.0074 for the converging and diverging channels, respectively.

The pressure at the inlet and outlet are prescribed by fixing their ratio and specifying a density. A constant pressure is implemented in the scheme by adjusting the particle density at the incoming link to the difference of the remaining links from the prescribed value according to its weight $w_{i}$. For example, at the inlet, the particle density of links 1, 5 and 8 (figure $1 a$ ) is kept higher than the links 3, 6 and 7 in order to induce a flow to the right (into the computational domain). A bounce-back boundary condition is used at the walls. This condition allows slip at the wall for high values of $K n$ (Nie et al. 2002). The flow starts from rest and is driven by the pressure gradient across the channel. The value of $K n$ is obtained from the pressure distribution. The Reynolds number $R e$ (based on the width of the narrower section and velocity at the centreline of the junction) and Knudsen number in the computation correspond to the range typically observed in microchannels. The results have been computed after a steady state is achieved such that the difference in mass flux across the channel is less than $0.05 \%$.

\section{Results}

\subsection{Pressure distribution}

The pressure distribution at the centreline for a given pressure ratio and different values of $K n$ for both the converging and diverging channels is shown in figure $2 a$. The pressure variation is nonlinear and shows a discontinuity in slope at the junction. The nonlinear pressure distribution in straight channels is due to the effects of compressibility and rarefaction (Beskok et al. 1996). The large pressure difference in the channel results in an expansion of the gas, leading to an acceleration of the flow. An accelerating flow however tends to reduce the pressure. These two opposing effects result in a nonlinear pressure distribution. The discontinuity in slope for the streamwise pressure is due to the effect of compressibility combined with the sudden change in cross-sectional area. This implies that for the present flow the information does not travel either upstream or downstream, which is at odds with macro flows 
where a perturbation is felt some distance away from it. The results for the different values of $K n$ imply that the deviation from a linear behaviour reduces with an increase in $K n$. This agrees with Beskok et al.'s (1996) observation for straight channels that compressibility and rarefaction have opposite effects, the former tending to increase the nonlinearity and the latter to reduce it.

Writing the pressure distribution in a series of microchannels as a combination of results from a single channel (Arkilic et al. 1998), we obtain

$$
\begin{aligned}
& \frac{P}{P_{j}}=-6 K n_{j}+\left(\left(6 K n_{j}+\frac{P_{i}}{P_{j}}\right)^{2}-\left(\left(\frac{P_{i}}{P_{j}}\right)^{2}-1+12 K n_{j}\left(\frac{P_{i}}{P_{j}}-1\right)\right) \frac{x}{L_{i}}\right)^{1 / 2}, \\
& \frac{P}{P_{o}}=-6 K n_{o}+\left(\left(6 K n_{o}+\frac{P_{j}}{P_{o}}\right)^{2}-\left(\left(\frac{P_{j}}{P_{o}}\right)^{2}-1+12 K n_{o}\left(\frac{P_{j}}{P_{o}}-1\right)\right) \frac{x}{L_{o}}\right)^{1 / 2}
\end{aligned}
$$

where $P$ is pressure, the subscripts $i, o$, and $j$ stand for inlet, outlet and junction respectively, and $L_{i}$ and $L_{o}$ are the length of the channel before and after the junction ( $L_{i}=L_{o}$ in our simulations). Note that (3.1) and (3.2) respectively represent pressure in the sections before and after the junction. These expressions are similar to those of Lee et al. (2002) but without the additional pressure loss term. We compare the pressure computed from these expressions to the simulation results. Figure 2(b) reveals an almost perfect agreement between the theoretical and simulated pressure distribution. Lee et al. (2002) measured a pressure drop across the junction, and attributed it to a loss during transition. The simulation suggests that there is no pressure loss at the junction; rather, the pressure drop is a natural consequence of the change in pressure corresponding to the change in cross-sectional area. In other words, fixing a probe slightly upstream and downstream of the junction will reveal a large pressure difference; but because the pressure in each section can be predicted well by theory, the pressure drop is entirely due to the change in cross-sectional area.

If the pressure ratio is held constant and the area ratio is varied (a maximum area ratio of 6 was tried) for diverging channels, it was found that the pressure in the narrow section of the channel of the smaller area ratio drops very rapidly. This is expected because of a relatively small pressure drop with a large area ratio in the wider section. The pressure remains nonlinear in both the narrow and wide channels and preserves the typical distribution of figure 2 .

\subsection{Velocity distribution and flow pattern}

The longitudinal velocity $U$ in a straight channel increases with $x$ (the streamwise coordinate) because of the expansion of the gas. (Note that all the velocities in this paper have been normalized by the speed of sound.) Further, $U$ is non-zero at the walls (because of a finite slip), and the slip velocity increases with $x$. However, the velocity profile remains parabolic. On the other hand, the lateral velocity $V$ is zero at the centreline (by symmetry) and at the wall (impervious wall) and exhibits maxima between these two locations (Arkilic et al. 1998; Lim et al. 2002). In other words, the effect of the expansion of the gas results in a maximum lateral velocity between the centreline and the walls, and there is a net movement of the gas towards the walls. Our simulations reproduce these features for both the wide and narrow sections of the channels away from the junction (figure 3). However, the flow profiles cannot be expected to exhibit this steady behaviour close to the junction (figure 3).

Surprisingly, the streamwise variation of $U$ shows a jump at the intersection of the channels (figure 4). (There seems to be some jitter at the beginning and end of the channel probably due to the pressure boundary condition. It can also be because of 

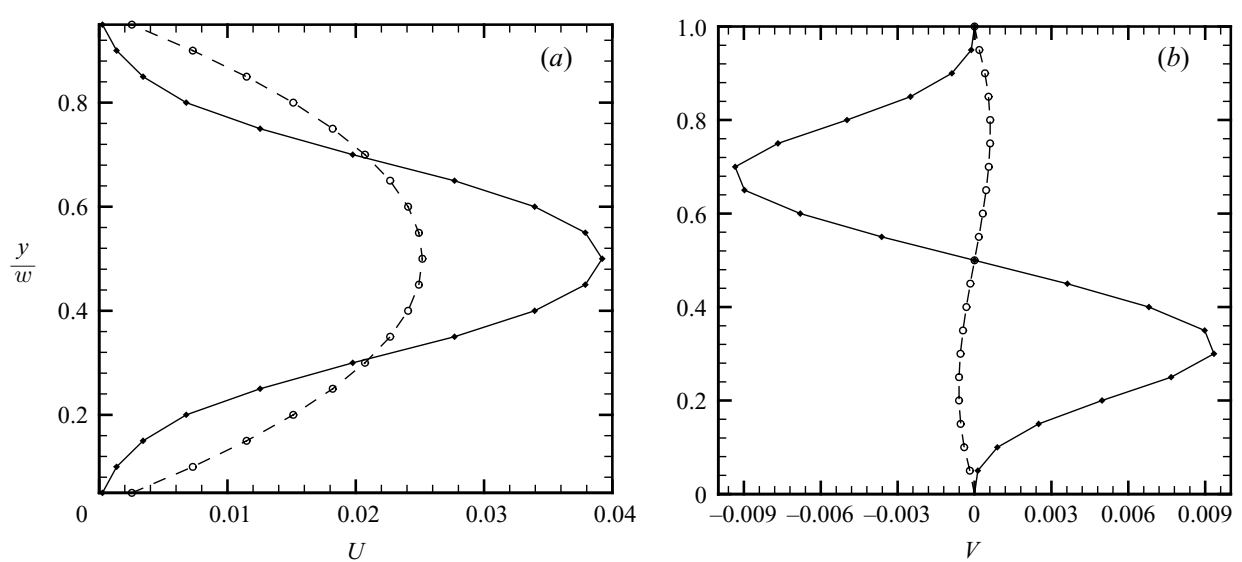

FIGURE 3. (a) Streamwise velocity profile at the centre of the wider section (dashed) and near the junction (solid) for the diverging channel. The $y$-axis has been normalized by the width, $w$, of the channel. (b) Lateral velocity profile for the corresponding locations (the lateral velocity at the centre has been multiplied by 10000 ).
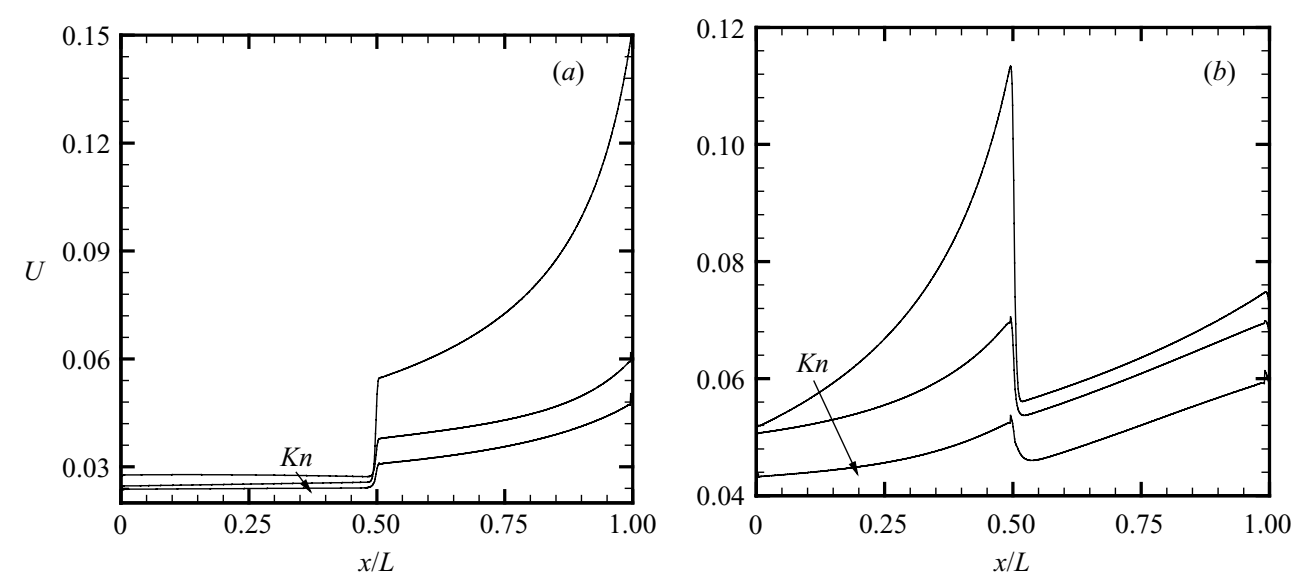

FIGURE 4. Streamwise velocity at the centre of $(a)$ the converging, and $(b)$ diverging channels for different values of $K n$. Note that some of the curves have been shifted upwards to facilitate the comparison. The $x$-axis has been normalized by the length $(L)$ of the channel.

entry and exit effects.) Figure 5 shows that there are sufficient points (more than 10) to represent this sharp rise or fall, and suggests that the flow rapidly adjusts to the change in cross-sectional area. The sudden change in velocity can be inferred from the continuity equation $\rho U A=$ constant. Because the variation in density $\rho$ is continuous, the velocity has to adjust to the change in area $A$. The jump in velocity differs from shocks in compressible macroflows because here the jump in velocity is not accompanied by a jump in pressure. A jump in velocity suggests that because of the rarefaction effect there is little communication between the different parts of the flow. Figure 5 further indicates that the change in $U$ is not symmetric with respect to the intersection point; instead, the change occurs mostly in the wider section. The result is surprising because there is no such jump in 'normal' channels. The difference appears to be related to the compressibility effect which plays an important role in microchannels. The jump in $U$ reduces as $K n$ increases for both converging and diverging channels, 


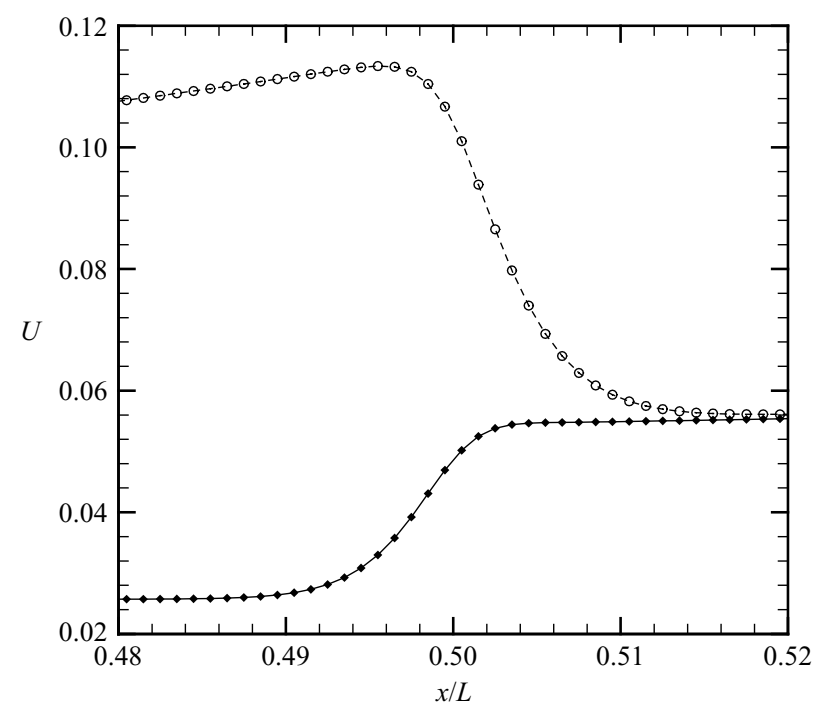

FIGURE 5. Magnified view of the streamwise velocity profile near the intersection of the channels (solid line: converging, dashed line: diverging channel).

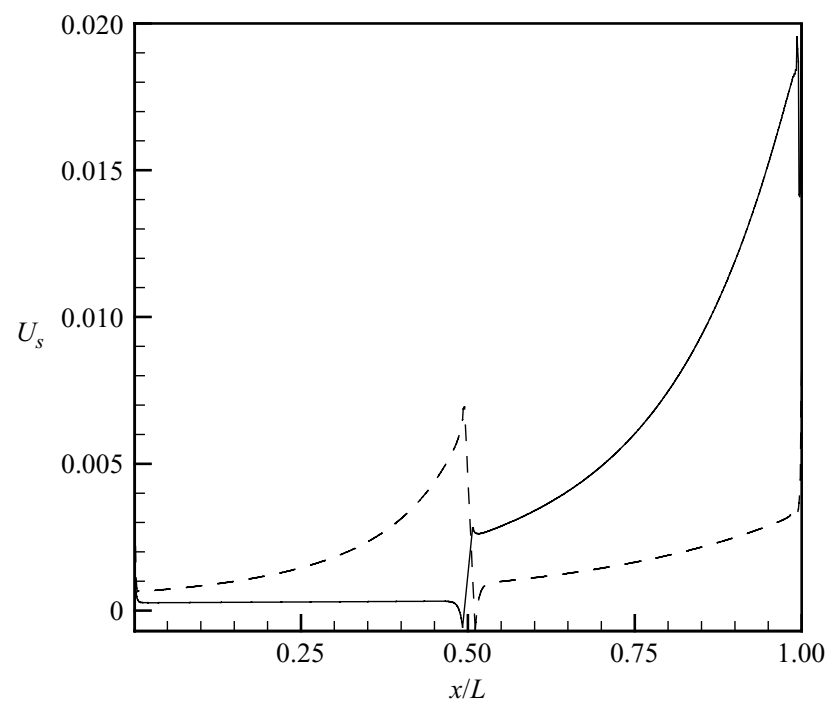

FIGURE 6. Slip velocity as a function of the streamwise coordinate (solid line: converging, dashed line: diverging channel).

i.e. compressibility and rarefaction have opposite effects as noted previously. The slip velocity $U_{s}$ increases with $x$ for both converging and diverging channels (figure 6) in a manner similar to figure 4 . Because $U_{s}$ is computed by fitting a parabola to the velocity profile which is not a good approximation near the intersection (figure $3 a$ ), $U_{s}$ shows a slight negative value (which is probably spurious) near the intersection. An important consequence of the slip is that for a given pressure ratio and area additional mass flux can be sustained through the channel (Karniadakis \& Beskok 2002).

Unlike normal channels, the flow in microchannels does not separate at the corners for the diverging geometry (figure $7 a$ ), and there is no vena contracta for the 

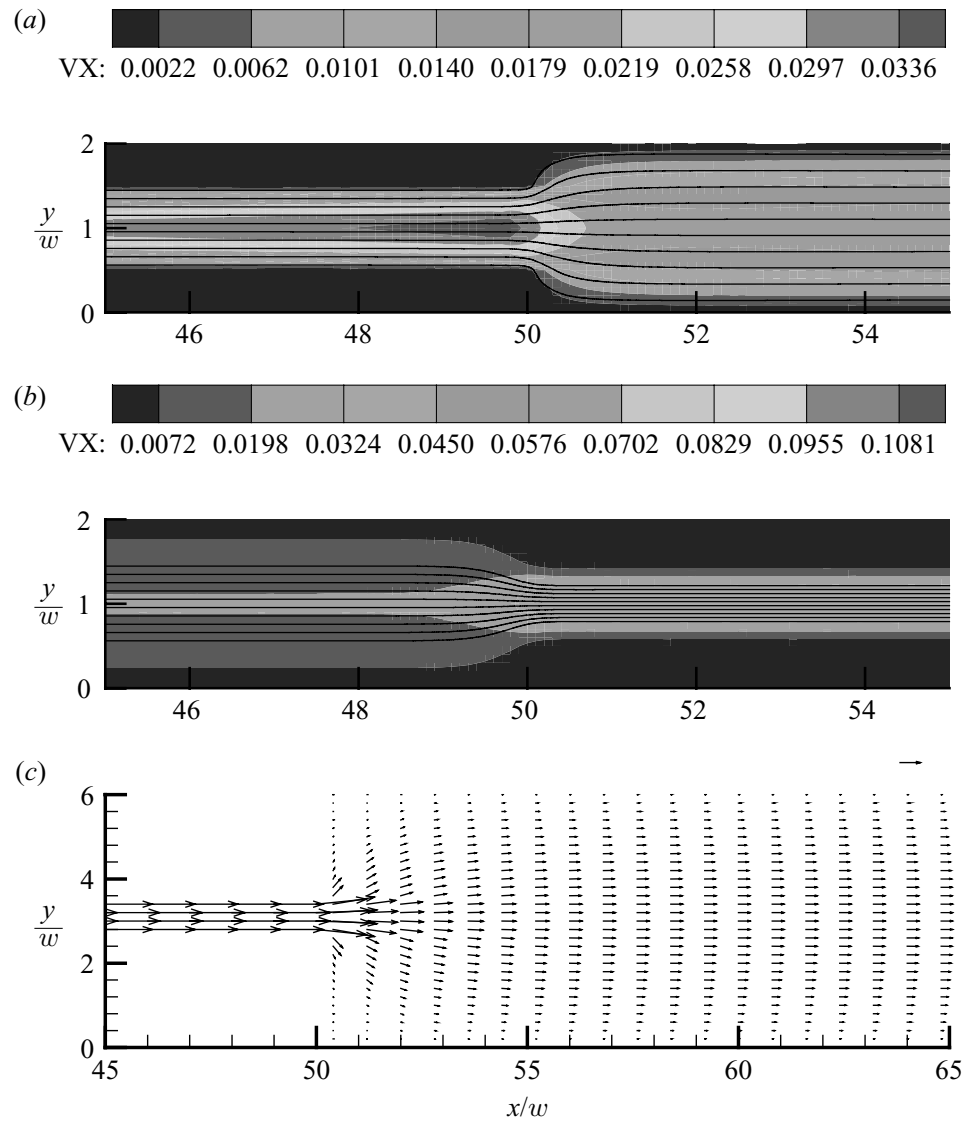

FIGURE 7. Streamlines for $(a)$ the diverging and $(b)$ converging channels with superposed contours of the streamwise velocity. The streamlines are reminiscent of potential flows. (c) Vector field for a wider area-ratio diverging channel. The number of vectors has been substantially reduced in order to avoid overcrowding the figure. The $x$ - and $y$-axes are normalized by the width $(w)$ of the channel. A reference vector of 0.003 times the speed of sound is also shown.

converging case (figure $7 b$ ). In other words, the streamlines are attached and the secondary losses are negligible in microchannels under conditions typically observed. Interestingly, these results apply even when the area ratio is increased substantially (up to 6) (figure 7c). The absence of vortex formation at the corner is probably because of slip which reduces the strength of vorticity generation at the wall. Lee et al. (2002) and Li et al. (2000) had conjectured that there are secondary pressure losses, based on experience with macroflows, to explain the large pressure drop in their measurements. As seen above, not only is the measured pressure drop due solely to the location of the measuring probes upstream and downstream of the junction, but there is no (additional) secondary pressure loss.

\subsection{Mass flow}

The mass flow can be easily computed once the velocity and density distributions in the channel are known. Almost the same amount of gas flows through the converging and diverging channels for a given pressure ratio (table 1). By keeping all parameters fixed, a slightly larger mass flux is obtained for a converging channel than a diverging 


$\begin{array}{ccllc}P_{i} / P_{o} & \text { Density } & R e_{\text {con }} & R e_{\text {div }} & M_{\text {con }} / M_{\text {div }} \\ 1.5 & 0.1 & 0.849 & 0.897 & 1.00 \\ & 0.03 & 0.0869 & 0.0889 & 1.02 \\ 2 & 0.01 & 0.0208 & 0.0203 & 1.04 \\ & 0.1 & 1.41 & 1.49 & 1.00 \\ 3 & 0.03 & 0.147 & 0.146 & 1.02 \\ & 0.01 & 0.0357 & 0.0334 & 1.07 \\ & 0.1 & 2.12 & 2.22 & 1.00 \\ & 0.03 & 0.224 & 0.216 & 1.04 \\ & 0.01 & 0.0517 & 0.0499 & 1.12\end{array}$

TABLE 1. Ratio of mass flux for the diverging to converging channels (with area ratio $=2$ ) for different densities (a larger density corresponds to a lower $K n$ ). $M$ is the mass flux, and subscripts 'div' and 'con' refer to the diverging and converging channel, respectively.

channel which suggests that the flow is not entirely reversible. Compared with macroflows, the energy loss is large for diverging channels because of the large secondary pressure losses, and, for a given pressure ratio, a much smaller mass flow can be sustained through a diverging channel compared to a converging channel. The result is in general agreement with the mass flow measurements of Lee et al. (2002). They also verified that the result holds for other included angles as well. Table 1 also tabulates the ratio at various values of particle density (or $\mathrm{Kn}$ ) and pressure ratio. Based on this information, we conclude that secondary losses are small in microchannels and perhaps other microdevices as well.

\section{Concluding remarks}

A numerical study has been undertaken on microchannels with a sudden expansion or contraction. These simulations are the first of their kind for complicated microdevices. Good agreement with the theory and experiments indicates that the simulations are effective in computing the flow through microdevices, and can therefore be undertaken for predicting the flow behaviour at a much lower cost and effort than experiments.

The pressure drop for both the converging and diverging channels shows a discontinuity in slope at the intersection of the channels. However, the pressure in each segment can be predicted well by the theory for straight channels. A jump in streamwise velocity accompanies the discontinuity in pressure drop, the strength of the jump being reduced as $K n$ increases. The cause of these discontinuities is attributed to compressibility effects. These are different from those in flows with shocks, where there is a discontinuity in both pressure and velocity across the shock. The results indicate that there is little transfer of information between different parts of the flow brought about by the rarefaction of the gas medium.

The amount of mass flow is almost the same for converging and diverging channels. The streamlines are attached even at the corners suggesting that they experience small secondary losses. From the results, it is also deduced that compressibility and rarefaction have opposite effects. Surprisingly, these results indicate that the complex channels of the type considered in this paper can be understood simply from a knowledge of results for their primary units, i.e. straight channels. This opens the possibility of understanding complex microchannels in terms of their primary 
units, which if conclusively shown will constitute a significant advancement in our understanding of a network of microchannels.

The continuing support of Australian Research Council (ARC) is gratefully acknowledged.

\section{REFERENCES}

Arkilic, E. B., Schmidt, M. A. \& Breuer, K. S. 1997 Gaseous slip flow in long microchannels. J. Microelectromech. Syst. 6, 167-178.

Beebe, D. J., Mensing, G. A. \& Walker, G. M. 2002 Physics and applications of microfluidics in biology. Annu. Rev. Biomed. Engng 4, 261-286.

Beskok, A., Karniadakis, G. E. \& Trimmer, W. 1996 Rarefaction and compressibility effects in gas microflows. J. Fluids Engng 118, 448-456.

Chen, S. \& Doolen, G. D. 1998 Lattice Boltzmann method for fluid flows. Annu. Rev. Fluid Mech. 30, 329-364.

Frisch, U., Hasslacher, B. \& Pomeau, Y. 1986 Lattice gas automata for the Navier-Stokes equations. Phys. Rev. Lett. 61, 2332-2335.

Ho, C. M. \& TAI, Y. C. 1998 Micro-electro-mechanical systems (MEMS) and fluid flows. Annu. Rev. Fluid Mech. 30, 579-612.

Karniadakis, G. E. \& BeskoK, A. 2002 Microflows - Fundamentals and Simulations. Springer.

LeE, W. Y., Wong, M. \& ZohaR, Y. 2002 Microchannels in series connected via a contraction/ expansion section. J. Fluid Mech. 459, 187-206.

Li, X., Lee, W. Y., Wong, M. \& Zohar, Y. 2000 Gas flow in constriction microdevices. Sensors Actuat. A 83, 277-283.

Lim, C. Y., Shu, C., Niu, X. D. \& Chew, Y. T. 2002 Application of lattice-Boltzmann method to simulate microchannel flows. Phys. Fluids 14, 2299-2308.

Meinhart, C. D., Wereley, S. T. \& Santiago, J. G. 1999 PIV measurements of a microchannel flow. Exps. Fluids 27, 414-419.

Nie, X., Doolen, G. D. \& Chen, S. 2002 Lattice-Boltzmann simulations of fluid flows in MEMS. J. Statist. Phys. 107, 279-289.

Pong, K., Ho., C. M., LiU, J. \& TAI, Y. C. 1994 non-linear pressure distribution in uniform microchannels. In Appl. Microfabrication to Fluid Mechanics, ASME Winter Annu. Meet., Chicago, Nov. 1994, pp. 51-56. 